\title{
Surface Characterization on Electrophoretic Deposition Oof 316I Stainless Steel with Dissolved Chitosan for Biomedical Application
}

Putu Hadi Setyarini

Mechanical Engineering Department Brawijaya University MT. Haryono, 167, Malang, INDONESIA putu_hadi@ub.ac.id

Femiana Gapsari Mechanical Engineering Department Brawijaya University MT. Haryono, 167, Malang, INDONESIA

Apollo Ode Rea Harjo
Mechanical Engineering Department
Brawijaya University
MT. Haryono, 167, Malang,
INDONESIA

Of many compounds materials, metallic biomaterial is widely used in human medical devices. An implant material's corrosion resistance impacts its flexibility and longevity. This corrosion resistance is also an important consideration for biocompatibility. This biomaterial contains stainless steel whih may corrode after being installed in the human body since the passivity of stainless steel disappears when it is exposed to acids in the human body for a long duration. In addition, lacks of oxygen inhibits the formation of a new $\mathrm{Cr}_{2} \mathrm{O}_{3}$ layer for corrosion protection. Based on this phenomenon, an advance treatment is required to improve the resistance corrosion of implant. This study investigates the effect of the concentration and duration of chitosan on the thickness and corrosion resistance of biomaterials. The concentration of chitosan used was $0.08 \%, 0.16 \%$, and $0.24 \%$ while the voltage used was constant at $10 \mathrm{~V}$. The test results demonstrated the lowest corrosion rate occrured for $0.24 \%$ chitosan concentration with 30 minutes coating duration. The lowest corrosion rate achieved was 0.014 mmpy and the maximum thickness was $75.4 \mu \mathrm{m}$. This study could be then used as a new solution to increase the safety of existing implants using biodegradable and non-toxic compounds. The next experiment should be implantation in real human body.

Keywords: Stainless Steel 316L, Chitosan, Electrophoretic Deposition

\section{INTRODUCTION}

A biomaterial is a substance which inactively interacts with the biological system [1-4]. Before the arrival of a new biomaterial into the market, numerous issues have been discussed, e.g. its anatomical position, tissue function, pathology composition, mechanical and other criteria for properties, toxicology, biocompatibility, healing mechanism, ethics, standardisation and regulatory procedures. International organizations shall issue guidelines on products, facilities and procedures of biomaterials to discourage inadequate testing of machinery and products and filter unqualified individuals in biomaterial production.

Biocompatibility considers the processes between hydrocarbons and the biomaterial itself. A biocompatible substance performs in a certain application with an acceptable host response, i.e. a minimal interruption to normal body function. Therefore, when put in vivo, the substance does not induce a thrombogenic, poisonous or allergic inflammatory reaction. For example, the biocompatibility of a drug is determined by two main factors, i.e. host reactions caused by this material and deterioration of the body environment. It is also important to weigh all variables since biomaterials appropriate for implants are a sufficiently corrosive medium to the human body. That is reasoning intensively selection of modern and effective biomaterials [5,6].

In the nature and selection of metals and alloys for in vivo use, corrosion is a major important factor. During corrosion cycles, allergens, harmful, hazardous or carcinogenic organisms may introduce into the human body. Various corrosion processes can also lead to the loosening and failure of implants [7]. Consequently, before they are licensed by governmental organisations, biomaterials should also be strictly screened for their corrosion resistance properties. For this purpose, the corrosion activity of metallic implant 
materials was extensively studied in the context of quality assurance, retrieval of an implant and malfunction investigation. Of many materials, the 316L stainless steel is widely used in biomedical application since this material has a high resistance to corrosion, excellent mechanical characteristics and relatively low production costs [8]. The surface oxide layer stability is one of the most important biomaterial properties [9]. Following electrolytic polishing, in the presence of physiological human body fluid conditions, the 316L stainless steel forms a very thin, i.e. few nanometer lightweight corrosion resistant film. The electrical deposition is generally recommended as a finishing procedure for metal implants in order that the elements which are a special covering substance are selectively dissolved [10]. Metals, polymers, ceramics and bioglass, or composite coatings may be co-deposited or formed layer after layer. Of various coating materials, chitosan is one of the materials commonly used as a coating [11]. This work aims to evaluate the surface characteristics of SS $316 \mathrm{~L}$ due to dissolved chitosan after the electrophoretic deposition process.

\section{MATERIALS AND METHODS}

\subsection{Coated specimen}

Table 1 shows the chemical composition of $316 \mathrm{~L}$ stainless steel used in this study. In this study, the discovered section or area used for corrosion evaluation on the specimen was $1 \mathrm{~cm}^{2}$.

Table 1: The chemical composition of SS 316L [\%Wt]

\begin{tabular}{c|c|c|c|c|c|c|c|c|c}
\hline $\mathbf{C}$ & $\mathbf{S i}$ & $\mathbf{M n}$ & $\mathbf{P}$ & $\mathbf{S}$ & $\mathbf{C r}$ & $\mathbf{N i}$ & $\mathbf{M o}$ & $\mathbf{N}$ & $\mathbf{F e}$ \\
\hline 0.023 & 0.46 & 1.81 & 0.031 & 0.001 & 17.1 & 10.1 & 2.03 & 0.032 & 68.413 \\
\hline
\end{tabular}

\subsection{Electrophoretic deposition process}

This analysis is searching for an optimal chitosan concentration. The chitosan concentrations varied at $0.08 \%$, $0.16 \%$ and $0.24 \%$. Meanwhile, a depositing duration of 30 minutes was used in all varied chitosan concentrations. Each chitosan concentration is accomplished with time variations when the lower corrosion rate is obtained, 10 minutes, 20 minutes and 30 minutes, at optimum chitosan level. The investigations used the following control variables: A $10 \mathrm{~V}$ voltage, applied to the DC power supply instrument, the anode with constant chemical composition and dimensian carbon rod, the $316 \mathrm{~L}$ stainless steel cathode, constant distance between the cathode to the anode at $2 \mathrm{~cm}$, the ambient temperature, constant $2 \%$ acetic acid as electrolyte solution. In the beginning, the electrolyte solution was made by combining $2 \mathrm{~mL}$ of acetic acid and $98 \mathrm{~mL}$ of distilled water in the breakers glass. After the solution was made, chitosan was added to the liquid according to the investigated concentration. As an example, $0.08 \mathrm{gr}$ of chitosan was added into the solution for $0.08 \%$ chitosan concentration. After the chitosan addition, the mixture was stirred until the solution thickened or changed its colour.

\subsection{Coating thickness test}

The coating thickness was determined by using an instrument called the Time TT260 Coating Thickness Tester. This instrument has been widely used to assess the coating thickness of ferrous or non-ferrous materials. This instrument calculated the data coating thickness by positioning the stylus for a few seconds. The resulted data of this instrument are average thickness, its standard deviation, minimum and maximum surface thickness.

\subsection{Corrosion test}

A potentiostat is an electrochemical analysis instrument used to determine the corrosion rate in this study. The potentiodynamic settings of the Metrohm Autolab PGSTAT $204 \mathrm{~N}$ were implemented for a potential range of -1.15 to $1.15 \mathrm{~V}$, a scan rate of $0.01 \mathrm{mV} / \mathrm{s}$ and a phase potential of $0.001 \mathrm{mV}$. The reference electrode is $\mathrm{Ag} / \mathrm{AgCl}$ electrode, the auxiliary electrode is platinum and the working electrode is chitosan-coated $316 \mathrm{~L}$ stainless steel. The corroding solution exposed area is $1 \mathrm{~cm}^{2}$ in scale. The corrosion test solution is Phosphate buffered saline (PBS) which is a widely used biochemical solution in biological science as a solvent. The PBS contained $8 \mathrm{~g} \mathrm{NaCl}, 0.2 \mathrm{~g} \mathrm{KCl}, 1.44 \mathrm{~g} \mathrm{Na}_{2} \mathrm{HPO}_{4}, 0.24 \mathrm{~g} \mathrm{KH}_{2} \mathrm{PO}_{4}$, and 1 litre of $\mathrm{H}_{2} \mathrm{O}$ diluent. The PBS as a solvent, owing to the quality of the nutrient glucose and inorganic salts, and phosphates' puffering ability, is frequently used in experiments using living cells. In this study, the amount of the PBS solution used was $50 \mathrm{ml}$ for each test.

\section{RESULTS}

Figure 1 shows the Tafel plots for all varied chitosan concentrations. As the chitosan concentration increases, the potential curve shifts to the left. The test found that the chitosan concentration of $0.24 \%$ resulted in the lowest corrosion rate (CR), i. e. 0.01486 mmpy (Table 2). The chitosan concentrations of $0.16 \%$ and $0.08 \%$ 
resulted in a corrosion rate of $0.11868 \mathrm{mmpy}$ and $0.14093 \mathrm{mmpy}$, respectively. The highest corrosion rate was 0.348 mmpy for an untreated specimen.

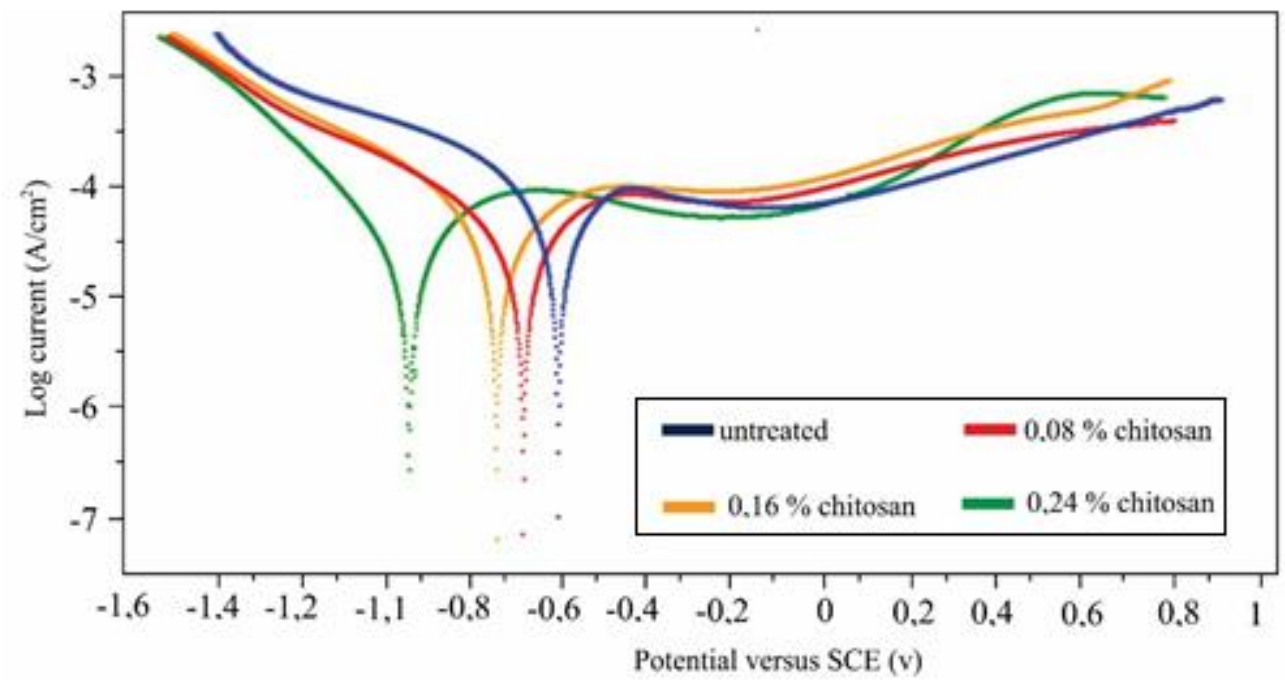

Figure 1: Tafel plots for the varied chitosan concentrations

Table 2 : Results of the influence of variance of the concentration of chitosan and the duration on the corrosion rate

\begin{tabular}{cccccc}
\hline $\begin{array}{c}\text { Chitosan } \\
\text { concen- } \\
\text { tration }\end{array}$ & Ba [mV/dec] & Bc [mV/dec] & $I_{\text {corr }}[\mu \mathrm{A}]$ & $E_{\text {corr }}[\mathbf{m V}]$ & CR [mmpy] \\
\hline Untreated & 855.41 & 4.886 & 33.591 & -454.16 & 0.34868 \\
$0.08 \%$ & 856.97 & 691.24 & 13.577 & -533.16 & 0.14093 \\
$0.16 \%$ & 412.75 & 996.68 & 11.433 & -593.34 & 0.11868 \\
$0.24 \%$ & 87.197 & 97.064 & 1.4321 & -792.58 & 0.01486 \\
\hline
\end{tabular}

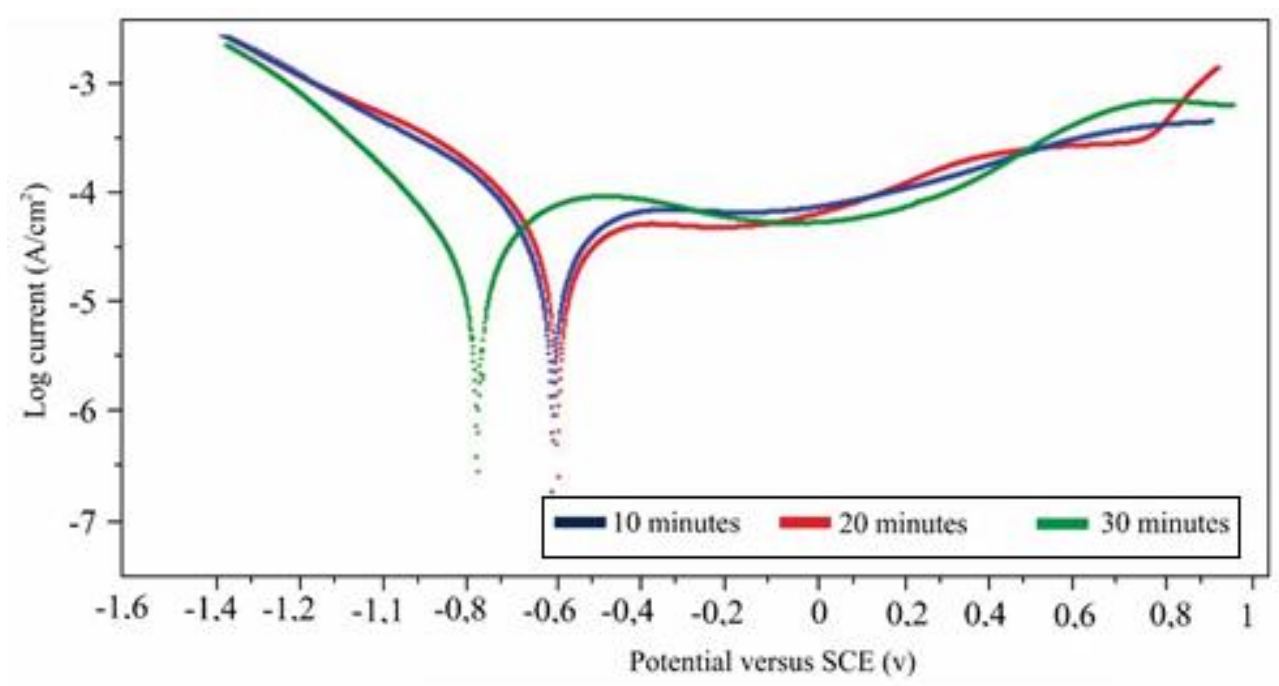

Figure 2: Current density for various coating duation of $0.24 \%$ chitosan concentration

This study found that the greater the chitosan concentration, the higher the corrosion resistance of the specimen which is due to the thicker chitosan layer deposited on surface of the $316 \mathrm{~L}$ stainless steel. To investigate this further, the coating duration was also checked against the corrosion rate (CR) from the optimum chitosan concentration. The effect of varied coating duration on the corrosion resistance for $0.24 \%$ chitosan concentration was calculated (Figure 2). Table 3 informs the corrosion resistance as a function of coating duration. The longest coverage life, i.e. at the lowest corrosion rate of $0.01486 \mathrm{mmpy}$, occurred 30 minutes coating duration. Following the mentioned corrosion rate, the corrosion rate of 20 and 10 minutes coating 
duration was 0.08860 and $0.10922 \mathrm{mmpy}$. The highest corrosion rate was for 10 minutes coating duration since the thinnest chitosan coating on the surface lead to the fastest contact between the corrosion-testing solution to the stainless steel surface.

Table 4 shows the influence of chitosan concentration increases on the coating thickness (Table 4). The coating duration was constant at 30 minutes for all chitosan concentrations. The lowest coating thickness of $15.1 \mu \mathrm{m}$ occurred for $0.08 \%$ chitosan concentration and the highest one, i.e. $75.4 \mu \mathrm{m}$, occurred for the highest chitosan concentration. As the dissolved chitosan quantity increases, deposition of chitosan on the cathode surface becomes easier. The data of coating thickness is in agreement with the corrosion rate, i.e. the lowest corrosion rate occurs for the thickest coating.

Table 3: Influence of coating duration on the corrosion rate for $0.24 \%$ chitosan concentration

\begin{tabular}{c|c|c|c|c|c}
\hline Duration [min] & Ba [mV/dec] & Bc [mV/dec] & Icorr $[\boldsymbol{\mu A}]$ & Ecorr [mV] & CR [mmpy] \\
\hline TP & 855.41 & 4.886 & 33.591 & $-454,16$ & 0.34868 \\
\hline 10 & 385.39 & 985.00 & 10.522 & -621.66 & 0.10922 \\
\hline 20 & 238.15 & 2.7953 & 8.5362 & -609.750 & 0.08608 \\
\hline 30 & 87.197 & 97.064 & 1.4321 & -792.58 & 0.01486 \\
\hline
\end{tabular}

Table 4: Influence of chitosan concentration on chitosan thickness

\begin{tabular}{c|c}
\hline Chitosan concentration [\%Wt] & Coating thickness [ $\boldsymbol{\mu m}]$ \\
\hline 0.08 & $15.1 \pm 0.258$ \\
\hline 0.16 & $21.5 \pm 1.161$ \\
\hline 0.24 & $75.4 \pm 6.649$ \\
\hline
\end{tabular}

Table 5: Effect of coating duration on thickness

\begin{tabular}{c|c}
\hline Duration [minutes] & Coating Thickness $[\boldsymbol{\mu m}]$ \\
\hline 10 minutes & $49.2 \pm 0.890$ \\
\hline 20 minutes & $51.6 \pm 2.969$ \\
\hline 30 minutes & $75.4 \pm 6.640$ \\
\hline
\end{tabular}

For $0.24 \%$ chitosan concentrate, coating thickness was observed as a function of coating duration (Table 5). The coating duration was observed for 10, 20 and 30 minutes. It is shown that a longer coating duration resulted in a thicker coating. The longer the coating duration, the more chitosan accumulated on the metal surface which increased the coating thickness. The thicker coating can effectively prohibit the interaction between the corrosion test solution and specimen surface. With an increase in average layer thickness, the average standard deviation obtained in this test has improved. The standard deviation indicates that the coating thickness is close to uniform. This is shown by a low standard deviation. With an increasing layer thickness, the standard deviation increases. This is due to the bubbles that appeared during the process of electrodeposition. Thus, uniform thickness becomes more difficult to be achieved.

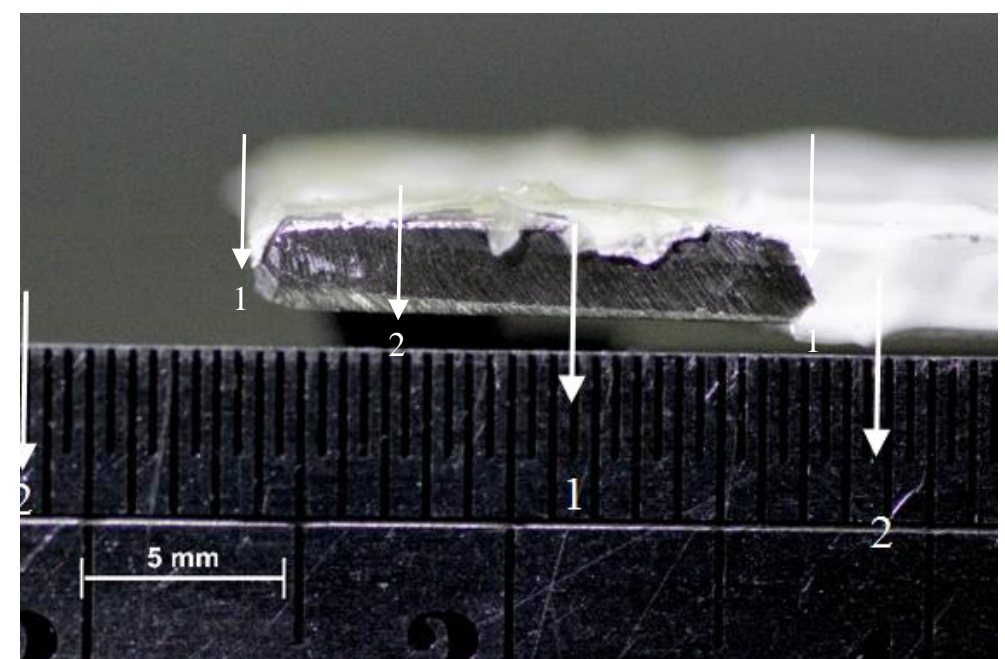

1: chitosan coating effects

2: $316 \mathrm{~L}$ stainless steel coating

Figure 3: Side view of chitosan-coated stainless steel

An exemplary macroscopic image of the coating result is shown in Figure 3. This figure is based on the 
thickest layer, i.e. $0.24 \%$ chitosan concentration and 30-minutes coating duration. A microscopic SEM analysis of $0.24 \%$ chitosan concentration and 30 minutes coating duration was worked out to provide visual investigation on the specimen surface (Figure 4). As previously discussed, the $0.24 \%$ chitosan concentration and 30 minutes coating duration results in the highest standard deviation, i.e. the highest range from the lowest to the highest thickness. The chitosan granules can be identified in Figure 4a and their magnification is depicted in Figure 4b. In addition to chitosan layer deposition, few cavities and pores are created during electrodeposition. This contributes to cavities formation on the surface.
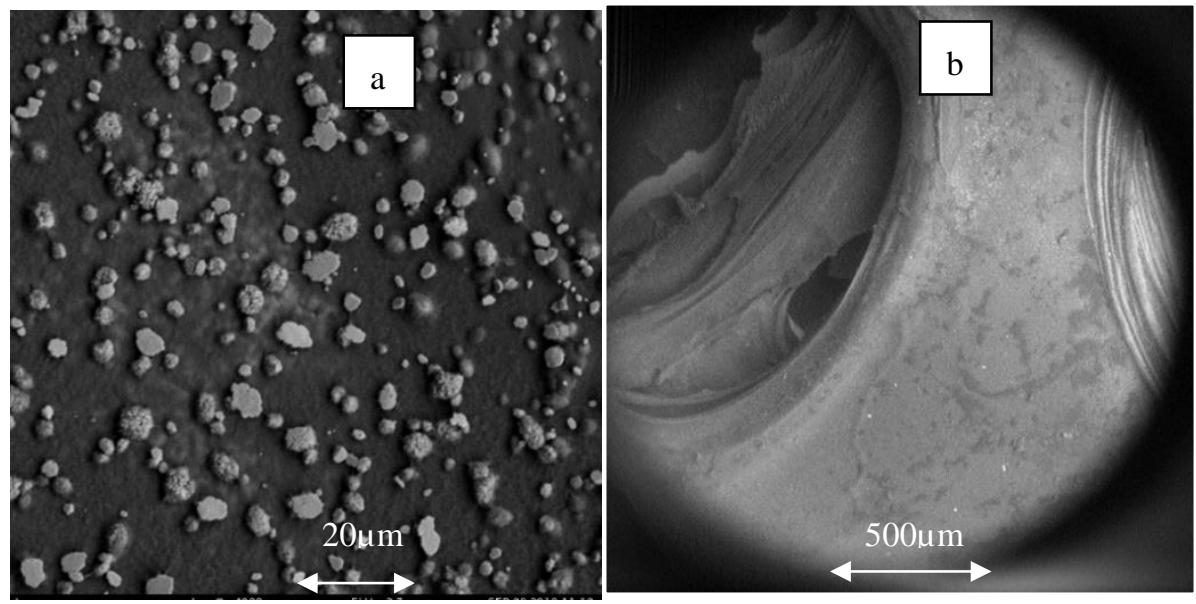

Figure 4: Microscopic photos of the surface of the layer after the electrodeposition process with a different magnification by SEM $a-4000 x ; b-400 x$

\section{DISCUSSION}

Chitosan will easily bind to surfaces that have a negative load and can rapidly interact with metal or metal oxide. It can form metal ion complexes and form polyanion gel. Chitosan is very easy to react with hydroxyl and amines. This material, therefore, is beneficial and ideal as a coating material. Since chitosan has many different hydroxyl $(\mathrm{O}-\mathrm{H})$ and amino $(\mathrm{N}-\mathrm{H})$ groups, it is therefore not harmful to the human body and can thus be used in the human body. In the electrophoretic deposition (EPD) under the influence of an electric field, filled particles or suspended polymers migrate toward the electrodes. The rate of specific migration during electrophoresis is affected by several factors, i.e. particle loading, the used electrical field, temperature and media properties. The EPD process occurs in 2 stages. In the first step, the particle suspension travels to the electrode following electrification. In the second step, the particles are placed on the electrodes to form a dense and uniform film surface. With the assistance of electric currents and a variety of chemical additives, the metal deposition process can be accomplished by the electrical deposition process to pass the coating metal to the coating substrate. The anode is an electrode attached to the positive pole at the DC power supply. An oxidation reaction occurs on the anode surface. The cathode is attached to the negative DC power supply and to the item to be coated. Meanwhile, the electrolyte solution is a medium that produces ions to superpose for conducting electricity between the cathode and the anode.

The method of EPD affects the physical and mechanical properties of coated materials. When an object is coated, the physical improvements are improved corrosion resistance and increased conductivity. The tensile and compressive strength of a substance after being coated is adjusted compared with before. The metal placing process commences with polarizing solvent molecules in the surrounding metal ions. Metal ions are formed from e electrolytes that dissolve metal anodes. The workpiece serving as a cathode is precipitated. There are various factors, e.g. duration, voltage, temperature, acidity and current, which influence the results. Longer coating duration results in higher coating thickness, i.e. the coating duration is directly proportional to the coating thickness since a longer coating duration increases the duration for coating elements to settle on the surface of coated material. Although higher voltage can easily increases the deposited substances, the consistency of the results of the deposited substances is much worse, e.g. non-uniform thickness. Layer deposition is a kinetic phenomenon. Thus, the created coating is influenced by the particle velocity. If the voltage used is larger, turbulent activity is induced so that the phase of deposition is disrupted by the current around the specimen. The higher the voltage, the greater the strain on the distribution and passage of particles. The electrical field influences the coating velocity and the composition of the coating. The coating process is also influenced by the temperature. Several variables, including the current used, and the distance between electrodes, affect the equilibrium temperature. Higher temperature decreases the solution viscosity. This leads 
to an increase in particle velocity in the solution. This rise in movement contributes to a faster formation pace leading to thicker coating. The $\mathrm{pH}$ of electrolyte solution used during the EPD process should be well controlled. During the process a neutral solution with a $\mathrm{pH}$ close to 7 , the solution is readily alkalinized on the cathode surface, mixing it up with alkaline salts or hydroxides. Meanwhile, an extreme $\mathrm{pH}$ or excessively acidic environment causes the hydrogen gas to emerge affecting the previously deposited material. The current density $\left[\mathrm{A} / \mathrm{cm}^{2}\right]$ also determines the efficiency of the coating. The cathode current density is the electric current required for each region of the specimen that must be overlain to obtain coating material atoms. The density of the current influences the thickness of the layer formed by electrical deposition.

The metal plating process commences with the surrounding metal ions creaated by the electrolytes or the dissolving metal anodes in electrolytes. The workpiece serving as a cathode induces precipitation. An area of the double electric layer (DEL), acting as a dielectric layer, is created near the cathode surface. Higher ions penetration occurs in the presence of the DEL layer. Metal ions travel to the cathode surface and absorb electrons from the cathode while positioning themselves on the cathode surface. The driving force for this process is the electrical potential difference assisted with chemical reactions.

In an equilibrium condition, ions are released onto the atoms from the cathode content. The contact between the three components, i.e. cathode steel, carbon anode and elecrolyte solution, is important for the coating process using chitosan. A $316 \mathrm{~L}$ stainless steel specimen is positioned the negative pole as the cathode. Carbon acts as an anode at the positive pole. Electrolyte containing acetic acid dissolves the chitosan at a determined concentration (eq. 1 and 2).

$\mathrm{CH}_{3} \mathrm{COOH}+\mathrm{H}_{2} \mathrm{O} \rightarrow \mathrm{CH}_{3} \mathrm{COO}^{-}+\mathrm{H}_{3} \mathrm{O}^{+}$,

Chit- $\mathrm{NH}_{2}+\mathrm{H}_{3} \mathrm{O}^{+} \rightarrow$ Chit- $\mathrm{NH}_{3}{ }^{+}+\mathrm{H}_{2} \mathrm{O}$.

Following reaction of each electrode occurs. The first reaction occurs when electrons from the anode flow to the cathode while the anode is converted to cathode by electrons. The second one occurs on the anode when oxygen contained in the electrolyte solution dissolves chitosan contains $\mathrm{O}_{2}$ molecules. In this process, water is decomposed (eq. 3).

$$
2 \mathrm{H}_{2} \mathrm{O} \rightarrow 4 \mathrm{H}^{+}+\mathrm{O}_{2}+4 \mathrm{e}^{-} \text {. }
$$

The anode used is a carbon rod that does not connect carbon ions to the surface of the coated workpiece as the electrons move from the anode to a cathode. Carbon anode settles on the bottom of the breaker glass during the process. In the meantime, $\mathrm{H}_{2} \mathrm{O}$ decomposition will occur at the cathode (eq. 4).

$$
2 \mathrm{H}_{2} \mathrm{O}+2 \mathrm{e}^{-} \rightarrow \mathrm{H}_{2}+2 \mathrm{OH}^{-} \text {. }
$$

If electrons pass from the anode to the cathode, the cathode raises electrons, and then chitosan-NH will result in the addition of electrons that attach to the surface of $316 \mathrm{~L}$ stainless steel (eq. 5).

$$
\text { Chit- } \mathrm{NH}_{3}{ }^{+}+\mathrm{OH}^{-} \rightarrow \text { Chit- } \mathrm{NH}_{2}+\mathrm{H}_{2} \mathrm{O}
$$

Chit- $\mathrm{NH}_{3}{ }^{+}$consists of Chit- $\mathrm{NH}_{2}$, which is eventually bound to the oxygen layer surface of stainless steel. This increases corrosion resistance by blocking the surface of the workpiece and surrounding environment by creating Chit- $\mathrm{NH}_{2}$ and $\mathrm{H}_{2} \mathrm{O}$.

The potentiodynamic polarization of 316L stainless steel displays the lowest corrosion risk occurs for $0.24 \%$ chitosan concentration with a coating duration of 30 minutes. Chitosan coating can minimize the current density of the 3161 stainless steel in PBS media. Time is one of the factors that affect the movement of particles in the EPD process. The increase in time duration causes the particles in the solution to move and stick more towards the electrode. From Table 5 it can be concluded that the longer the time applied in the EPD process, the thicker the deposited layer is. The corrosion current density obtained for 30 minutes coating duration has the smallest value and this is in agreement with the thickness test results since the layers formed are relatively uniform leading to the better resistance to corrosion. Meanwhile, at 10 and 20 minutes, the density of corrosion currents was quite high, because the formed layer was not uniform, and the layer formed was thinner. During the corrosion measurement process, the chloride ions contained in the phosphate buffer saline electrolyte are concentrated in the thinner layer. So that pitting corrosion can occur. These results indicate that the composite deposit could act as a protective layer and improve the corrosion resistance of the stainless steel substrate in the environment.

The uniformity of the created layers can be seen from the standard deviation value. Based on the standard deviation value the longer the coating time, the greater the standard deviation value, meaning that the longer the coating time, the more non-uniform the formed layer. The decrease in uniformity of this layer is caused by the coating formed on the electrode surface. At constant stress, the longer the EPD time, a thicker layer is formed. This layer formed acts as a barrier layer which decreases the current density between the two electrodes during the deposition process. The decrease in current density causes the movement of chitosan particles towards the electrodes to decrease. 


\section{CONCLUSIONS}

This study informs the influence of varied chitosan concentration and coating duration influences on the corrosion rate of biomaterials made of the $326 \mathrm{~L}$ stainless steel coated with chitosan. The lowest corrosion rate of $0.01486 \mathrm{~mm}$ occurred for $0.24 \%$ chitosan concentration and coating duration of 30 minutes. $\mathrm{N}$ agreement, the thickest layer of $75.4 \mu \mathrm{m}$ also occurred for $0.24 \%$ chitosan concentration and coating duration of 30 minutes. Meanwhile, the highest corrosion rate at 0.34868 mmpy occurred for the non-coated specimen. This study shows that longer deposition duration and higher chitosan concentration results in higher coating thickness layer. Meanwhile, the highest standard deviation of coating thickness, informing less homogenous coating, occurred for 30 minutes coating duration and $0.24 \%$ chitosan concentration.

\section{REFERENCES}

[1] HE, W., REAUME, M., HENNENFENT, M., LEE, B.P., RAJACHAR, P., "Biomimetic Hydrogels With Spatial- and Temporal-Controlled Chemical Cues for Tissue Engineering", Biomaterials Science, v. 8, pp. 3248-3269, May. 2020.

[2] XUEli, Z., SHUANG-QUAN, Z., XIAOYUAN, C., "Stereospecific Interactions Between Chiral Inorganic Nanomaterials and Biological Systems”, Chemical Society Reviews, v. 49, pp. 2481-2503, Mar. 2020.

[3] BISWAL, T., JENA, S.K.B., PRADHAN, D., "Sustainable Biomaterials and Their Applications: A Short Review", Materials Today: Proceedings, v. 30, n.2, pp. 274-282, 2020

[4] ZHU, L., LUO, D., LIU, Y., "Effect of The Nano/Microscale Structure of Biomaterial Scaffolds on Bone Regeneration", International Journal Of Oral Science, 12, article number : 6, Feb. 2020.

[5] YADAV, D., GARG, R.K., AHLAWAT, A., CHHABRA, D., "3D Printable Biomaterials For Orthopedic Implants: Solution For Sustainable And Circular Economy”, Resources Policy 68, 101767, Oct. 2020.

[6] ELIAZ, N., "Corrosion Of Metallic Biomaterials: A Review”, Materials, v. 12, n. 3, 407, Jan. 2019

[7] TALHA, M., MA, Y., KUMAR, P., YUANHUALIN, Y., SINGH, A., "Role of Protein Adsorption In The Bio Corrosion of Metallic Implants - A Review”, Colloids and Surfaces B: Biointerfaces, v. 176, pp. 494-506, Apr. 2019

[8] KIM, D.K., WOO, W., KIM, E.Y., CHOI, S.H., "Microstructure and Mechanical Characteristics of Multi-Layered Materials Composed of 3161 Stainless Steel and Ferritic Steel Produced by Direct Energy Deposition", Journal of Alloys and Compounds, v. 774, pp. 896-907, Feb. 2019.

[9] REVIE R., UHLIG H.H., Corrosion And Corrosion Control: An Introduction to Corrosion Science and Engineering. 4th Ed. New York, John Wiley \& Sons; Hoboken, USA, 2008.

[10] MENG, E.C., GUAN, S.K., WANG, H.X., WANG, L.G., ZHU, S.J., HU, J.H., REN, C.X., GAO, J.H., FENG, Y.S., "Effect Of Electrodeposition Modes on Surface Characteristics and Corrosion Properties of Fluorine-Doped Hydroxyapatite Coatings on Mg-Zn-Ca Alloy”, Applied Surface Science, v. 257, pp. 4811-4816, Mar. 2011

[11] PANG, X., ZHITOMIRSKY, I., "Electrodeposition Of Hydroxyapatite-Silver-Chitosan Nanocomposite Coatings", Surface Coating Technology, v. 202, n. 16, pp. 3815-3821, May. 2008. 